\title{
REVISÃO INTEGRATIVA DOS MARCADORES MOLECULARES DA LEUCEMIA LINFÓIDE CRÔNICA (LLC)
}

\author{
Nilson José Frutuoso da Silva, Lidiane Régia Pereira Braga de Britto Laboratório de \\ Biologia Celular e Molecular da Universidade de Pernambuco-Campus Petrolina
}

\author{
nilson.frutuoso@upe.br
}

\begin{abstract}
RESUMO
A leucemia linfoide crônica (LLC) é uma linfocitose monoclonal de linfócito B, com proliferação e acúmulo de linfócitos maduros no sangue periférico, MO e tecidos linfóides. Os biomarcadores moleculares são usados atualmente no processo de estudo de estratificação clínica, uma confirmação de um diagnóstico exclusivo; fornecendo informações prognósticas que apresentam fatores preditivos de resposta ao tratamento. Cerca de $80 \%$ dos prognósticos da LLC estão relacionadas às alterações genéticas, destacando-se quatro anormalidades cromossômicas: deleção de 13q14 (bom prognóstico), trissomia 12 (intermediário), deleção del1q23 (desfavorável), del 17p (ruim ao prognóstico). Por meio das alterações citogenéticas têm sido correlacionados ao prognóstico da LLC, tornando-se de grande relevância na identificação de alterações cromossômicas que possibilita estabelecer subgrupos de indivíduos com prognóstico diferenciado. Objetivo deste artigo é fazer pesquisa de revisão de integrativa sobre marcadores moleculares genéticos relacionados ao prognóstico no tratamento da LLC.
\end{abstract}

Palavras-chave: leucemia linfóide crônica; mutações cromossômicas; biomarcadores.

\section{INTRODUÇÃO}

As leucemias são um dos três principais tipos de cânceres que acometem o sangue (Koohi et al., 2015), sendo um grupo de doenças hematológicas, malignas, metastáticas e clonais dos linfócitos (Santos, 2013; Koohi et al., 2015) que se acumulam no sangue, medula 
óssea (MO) e tecidos linfóides. Onde podem ser classificadas como mielóide (ou mielocítica) e linfóide (ou linfocítica) e podem ser subclassificadas como aguda ou crônica (Montserrat; Moreno, 2008).

$\mathrm{Na}$ busca de exames na tentativa de associar diagnóstico e prognóstico da doença (Ruiz et al., 2010; Gonçalves et al., 2009; Metze, 2005) as indicações para um estudo citogenético numa suposição ou definitivo diagnóstico de neoplasias hematológicas, incluem proporcionar evidências de clonalidade, confirmar um diagnóstico exclusivo, permite definir a neoplasia, fornece informações ao prognóstico indicando na qual os genes de fusão é suscetível a estarem presente (Bain et al., 2012), assim podendo determinar subgrupos de pacientes ao prognóstico diferente (Chauffaille et al.,2006).

Cerca de $80 \%$ do prognóstico da LLC são relacionadas às alterações genéticas, destacando-se quatro anormalidades cromossômicas mais frequente: deleção de 13q14 (bom prognóstico), trissomia 12 (intermediário), deleção de 11q23 (desfavorável), del 17p (muito desfavorável ao prognóstico) (Chauffaille et al., 2006). O sistema de estadiamento clínico Rai e Binet (Rai et al., 1975; Binet et al., 1981) possibilita análise do tamanho da carga tumoral, previsão da evolução e/ou classificação dos pacientes que precisam do tratamento precoce (Chauffaille, 2005).

Faz-se necessário a realização dos estudos citogenéticos nos presentes casos de LLC e nas diversas fases evolutivas, a partir do diagnóstico. A citogenética clássica e, sobretudo a molecular, desempenham um papel fundamental a proporção que auxiliam na identificação de mutações genômicas das células malignas, classificando pacientes com mau prognóstico (desfavorável), distinguindo que receberá maior atenção e planejamento terapêutico (Chauffaille, 2005). Devido a este fato, é interessante realizar uma revisão na literatura sobre os marcadores genéticos já descritos com associação ao tipo de prognóstico para a LLC.

\section{METODOLOGIA}

A metodologia utilizada trata-se de revisão da literatura buscando publicações relacionadas a marcadores moleculares ao prognóstico. A busca foi realizada nas bases de dados eletrônicas nacionais e internacionais, periódico capes, PubMed e 
Scielo, utilizando as seguintes estratégias de busca (chronic lymphoid leukemia AND chomosomal mutation), (chronic lymphoid leukemia AND biomarkers), (chronic lymphoid leukemia AND prognosis), (chronic lymphoid leukemia AND molecular markers). Como a proposta da presente revisão integrativa é sintetizar os avanços relacionados ao tema foram considerados na seleção artigos científicos durante o ano de 2018 a setembro de 2021. Utilizando-se os filtros nos sistemas de busca, tendo como parâmetro de inclusão dos artigos: texto na íntegra, idiomas (português, espanhol e inglês) e ano de publicação. Em seguida seus títulos foram lidos, e aqueles que destoassem do tema da pesquisa, além de idiomas diferentes, foram eliminados da amostra. Em segundo momento foram lidos resumos e leitura na íntegra considerando a inclusão na revisão dos artigos que mantiveram pertinência ao tema proposto. Nesse momento foram excluídos artigos que não abordavam temas de interesse deste estudo.

\section{RESULTADOS E DISCUSSÕES}

Tabela 1- Alteração cromossômica comuns na LLC.

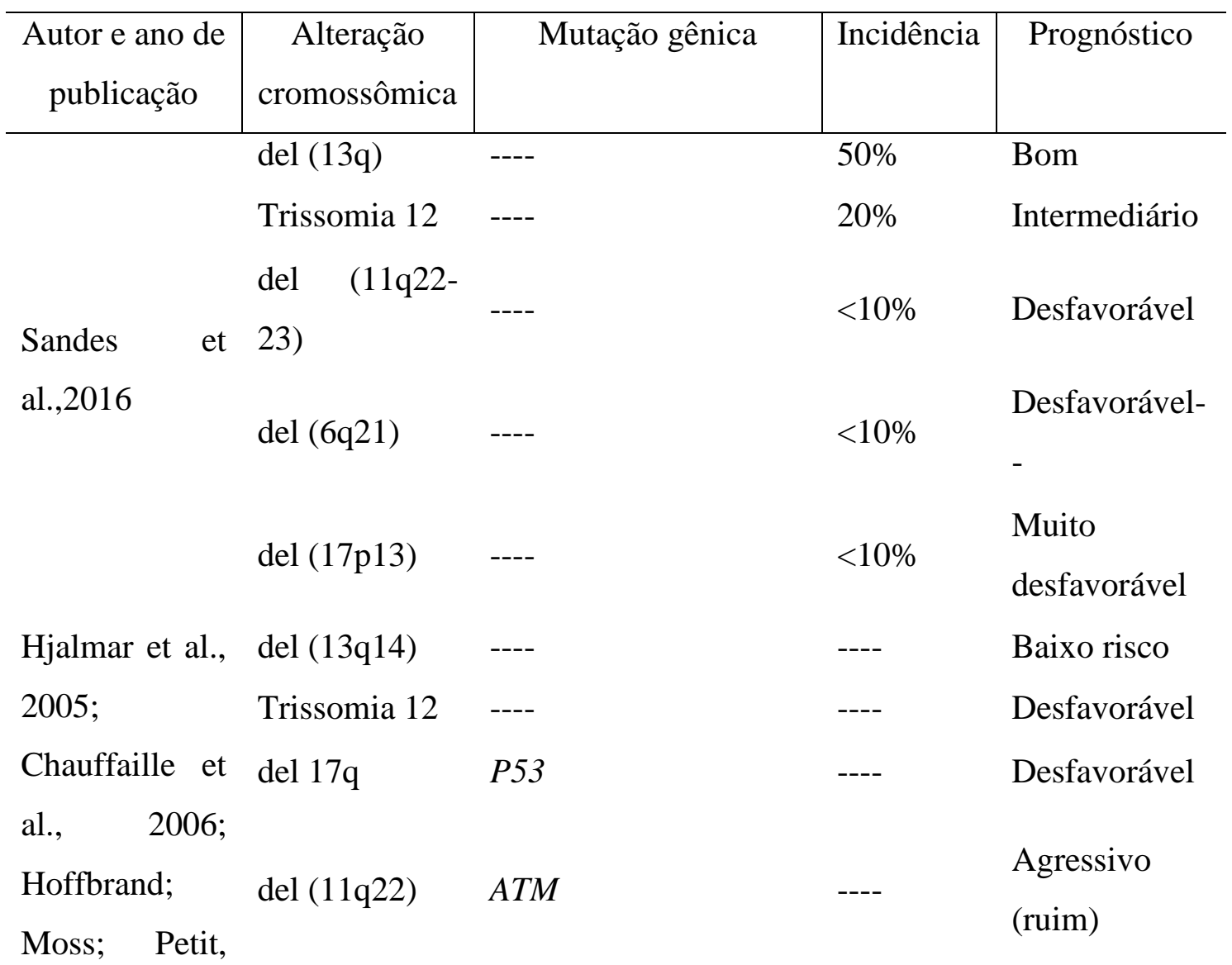


2008;

Hamerschlak, 2010

\begin{tabular}{|c|c|c|c|}
\hline del (13q14) & ---- & $50 \%$ & $\begin{array}{l}\text { Progressão } \\
\text { estável }\end{array}$ \\
\hline
\end{tabular}

Trissomia 12

MIR, 2016

\begin{tabular}{|c|c|c|c|c|}
\hline & $\operatorname{del} 17 q$ & P53 & ---- & $\begin{array}{l}\text { rápida/ curta } \\
\text { remissão }\end{array}$ \\
\hline & del (11q22) & ---- & $19 \%$ & Agressiva \\
\hline & $\operatorname{del}(13 q)$ & $\mathrm{miR} 15 / \mathrm{miR} 16$ & $55 \%$ & ---- \\
\hline Valdespino- & Trissomia $12 q$ & ---- & $7 \%$ & ---- \\
\hline \multirow[t]{2}{*}{ Gómez,2014 } & $\operatorname{del} 17 q$ & P53 & $16 \%$ & Mau \\
\hline & $\operatorname{del}(11 q)$ & & $18 \%$ & Mau \\
\hline $\begin{array}{l}\text { Cimmino et } \\
\text { al., } 2005\end{array}$ & del13q14) & miR15/miRl6 & $50 \%$ & Favorável \\
\hline $\begin{array}{l}\text { Winkler et al., } \\
2005\end{array}$ & Trissomia 12 & $\begin{array}{l}P 27, \quad C D K 4, \quad H I P 1 R, \\
\text { MYF6 е MDM2 }\end{array}$ & $10-20 \%$ & Intermediário \\
\hline $\begin{array}{l}\text { Stankovic et } \\
\text { al., } 1999\end{array}$ & del11q22q-23 & $A T M$ & $5-20 \%$ & Desfavorável \\
\hline $\begin{array}{l}\text { Gaidano et al., } \\
2012\end{array}$ & del17p13 & TP53 & $10 \%$ & Mau \\
\hline Dohner et al., & del11q & ---- & ---- & Mau \\
\hline 2000 & $\operatorname{del} 17 q$ & ---- & ---- & Mau \\
\hline \multirow{3}{*}{$\begin{array}{l}\text { Puiggros et al., } \\
2014\end{array}$} & $17 p$ & TP53/ BICR3 & ---- & $\begin{array}{l}\text { Altíssimo } \\
\text { risco }\end{array}$ \\
\hline & $11 q$ & ATM/NOTCHI/SF3B1 & ---- & Alto risco \\
\hline & Trissomia 12 & ---- & ---- & $\begin{array}{l}\text { Risco } \\
\text { intermediário }\end{array}$ \\
\hline
\end{tabular}

Fonte: Silva, N. J. F. 


\section{CONSIDERAÇÕES FINAIS}

Até o momento, a alteração cromossômica mais prevalente na literatura foi a deleção 13q, trissomia 12 , deleção $11 \mathrm{q}$, deleção $17 \mathrm{p}$ correspondente ao prognóstico favorável para a LLC.

\section{REFERÊNCIAS}

ABBOTT, B. L. Chronic lymphocytic leukemia: recent advances in diagnosis and treatment. The Oncologist. Colorado, v.11, p.21-30. 2006.

BAIN, B. J.; et al. Dacie and lewis practical haematology. 11. ed. China: Elsevier, p. $668,2012$.

BINET, J.L.; AUQUIER, A.; DIGHIERO, G.; et al. A new prognostic classification of lymphocytic leukemia derived from a multivariante survival analysis. Cancer; 48(1):198- 206, 1981.

CHAUFFAILLE, M. L. L. F. Citogenética e biologia molecular em leucemia linfocítica crônica. Rev. Bras. Hematol. Hemoter. v. 27 n. 4, p. 247-252, 2005.

CHAUfFAILlE, M. L. L. F.; et al. CLL: Chromosomal abnormalities (FISH) and their relation with clinical stage, CD38 and Zap-70. Revista Brasileira de Hematologia e Hemoterapia, São Paulo, v. 28, n. 1, p. 5-10. 2006.

CHAUFFAILLE, M. L. L. F; VIEIRA, S.; MARTINS, S. L. R. Importância do cariótipo em leucemia linfocítica crônica: relato de 18 casos. Jornal Brasileiro de Patologia e Medicina Laboratorial. São Paulo, v.40, n.2, p.75-78, 2004.

CHIATTONE, C.S. Indicação para o início de tratamento na leucemia linfoide crônica. Rev. Bras. Hematol. Hemoter. v. 27 n. 4. p. 272-275, 2005 a. 
CIMMINO, A.; et al. miR-15 and miR-16 induce apoptosis by targetting BCL-2. Proc. Natl. Acad. Sci. U. S. A. 102 (39), 13944-13949, 2005.

DOHNER, P.; et al. Genomic aberrations and survival in chronic lymphocytic leukemia. N. Engl. J. Med. 343 (26), 1910-1916, 2000.

GABE, C.; ALMEIDA, D. R.; SIQUEIRA, L. O. Evaluation of opportunistic infections in childeren suffering from leukemia. Rev. Bras. Hematol. Hemoter, Passo Fundo, v. 31, n. 2, p. 74-79. 2009.

GAIDANO, G.; FOA, R.; DALLA-FAVERA, R. Molecular pathogenesis of chronic lymphocytic leukemia. J. Clin. Invert. 122 (10), 3432-3438, 2012.

GARICOCHEA, B. Patogênese da leucemia linfóide crônica. Rev. Bras. Hematol. Hemoter. v. 27 n. 4, p. 241-246, 2005.

GARLEY, M.; et al. Expression of IL-1 and their natural regulators in leukocytes of B cell chronic lymphocytic leukaemia patients. Advances in Medical Sciences, Bialystok, v. 61, n.2, p. 187-192. 2016.

GONÇALVES, R. P.; et al. Avaliação do perfil hematológico de pacientes com leucemia linfocítica crônica (LLC-B) em um hemocentro estadual. Rev. Bras. Hematol. Hemoter. v. 31 n. 4, p. 228-234, 2009.

HAMERSCHLAK, N. Manual de hematologia: programa integrado de hematologia e transplante de medula óssea. 1. ed. Barueri: Manole, p. 531, 2010.

HJALMAR, V. Sequential fluorescence in situ hybridization analysis for trisomy 12 in Bcell chronic lymphocytic leukemia. Methods in Molecular Medicine. Totowa, v.115, p.231-240. 2005. 
HOFFBRAND, A. V.; MOSS, P. A. H.; PETIT, J. E. Fundamentos em hematologia. 5. ed. Porto Alegre: Artmed, p.400, 2008.

HOFFBRAND, A. V.; MOSS, P. A. H. Fundamentos em Hematologia. 6.ed. São Paulo: Artmed, p. 2-14, 2013.

JEMAL, A.; SIEGEL, R.; XU, J.; WARD, E. Cancer statistics, 2010. CA Cancer J Clin; 60:277-300, 2010.

KEATING, M. J. Chronic lymphocytic leukemia. In: ES Henderson, TA Lister \& MF Greaves. Leukemia 7th edition, Saunders CO, Philadelphia, p 656, 2002.

KERSTING, S.; et al. Dutch for the diagnosis and treatment of chronic lymphocytic leukemia. The Netherlands Journal of Medicine, Amsterdam, v.74, n.2, p.68-74, feb. 2016.

KOOHI, Fatemeh; et al. Leukemia in Iran: epidemiology and morphology trends. Asian Pacific Journal of Cancer Prevention, Iran, V. 16, n. 17, p. 7759-7763. 2015.

METZE, I. L. LLC: Critérios diagnóstico, imunofenotipagem e diagnóstico diferencial. Rev. Bras. Hematol. Hemoter. v. 27 n. 4, p. 233-235, 2005.

MIR, M. A. Chronic lymphocytic leukemia: practice essentials, pathophysiology, etiology. Medscape Internal Medicine. Atualizado: 07 de setembro de 2016.

MONTSERRAT, E.; MORENO, C. Chronic lymphocytic leukaemia: a short overview. Annals of Oncology. Barcelona, v. 19, p. 320-325, sept. 2008.

MULLER-HERMELINGK, H. K.; et al. Chronic lymphocytic leukaemia/small lymphocytic lymphoma. In: Swerdlow SH, Campo e, Harris NL, Jaffe 
ES, Pileri SA, Stein H, Thiele J, Vardiman JM, editors. WHO classification of tumours of hematopoietic and lymphoid. 4th ed. Geneve: WHO Press, p.180-182, 2008.

OLIVEIRA, R. A. G.; NETO, A. P. Anemias e leucemias: conceitos básicos e diagnóstico por técnicas laboratoriais. 1. ed. São Paulo: Roca, p. 421, 2004.

PDQ Adult treatment Conselho Editorial. PDQ leucemia linfocitica crônica tratamento. Bethesda, MD: National Cancer Institute. Atualizado: 28 de julho de 2016.

PUIGGROS, A.; BLANCO, G.; ESPINET, B. Genetic abnormalities in chronic lymphocytic leukemia: where we are and where we goll. Biomed Res. Int. 2014.

RADAELLI, A.; et al. The clinical ande epidemiological burden of chronic lymphocytic leukemia. Eur. J. Cancer Care; 13:279-87, 2004.

RAI, K. R.; et al. Clinical staging of chronic lymphocytic leukemia. Blood; 46(2):219234, 1975.

RIBEIRO, A. A. R. Leucemia linfocítica crônica B- A importância de deleção 17p. Porto. Universidade do Porto; 2010.

ROSSI, D.; et al. Disruption of BRC3 associated with fludarabine chemo refractoriness in TP53 wild-type chronic lymphocytic leukemia. Blood 119 (12), 2854-2862, 2012a.

RUIZ, M.A. et al. O transplante de célula-tronco hematopoéticas na leucemia linfoide crônica, uma proposta do I Encontro de Diretrizes do Transplante de Medula Óssea da Sociedade Brasileira de Transplante de Medula Óssea, Rio de Janeiro 2009. Rev Bras. Hematol. Hemoter. v. 32 Supl. 1, p. 91-96, 2010.

SÁNCHEZ, M. A. O.; ORTEGA, M. L. O.; BARRIENTOS, J. V. R. Leucemia linfoblástica aguda. Medicina Interna de México, Pachuca, v. 23, n. 1, p. 26-33. 2007. 
SANDES, Alex Freire; et al. Diagnóstico em hematologia. 1. ed. Barueri: Manole, p. $358,2016$.

SANTOS, P. C. J. L. (Coord.). Hematologia: métodos e interpretação. 1. ed. São Paulo: Roca, p. 450, 2013.

SCARFÒ, L.; FERRERI, A. J. M.; GHIA, P. Chronic lymphocytic leukaemia. Critical Reviews in Oncology/Hematology. Italy, v.104, p.169-182, 2016.

SCHWARTZ, G. G.; KLUG, M. G. Incidence rates of chronic lymphocytic leukemia in US states are associated with residential radon levels. Future Oncology, USA, v. 12, n. 2, p. 165-174. 2016.

STANKOVIC, T.; et al. Inactivation of ataxia telangiectasia mutated gene in Bcell chronic lymphocytic leukaemia. Lancet 353 (9146), 26-29, 1999.

STILGENBAUER, S.; BULLINGER, L.; LICHTER, P.; DOHNER, H. Genetics of chronic lymphocytic leukemia: genomic aberration and $\mathrm{V}(\mathrm{H})$ gene mutation status in pathogenesis and clinical course. leukemia. 16(6):993-100, 2002.

STRATI, P.; et al. Prevalence and Characteristics of Central Nervous System Involvement by Chronic Lymphocytic Leukemia. Haematologica, Chicago, p. 124. 2016.

VALDESPINO-GÓMEZ V. M. Leucemia linfocítica crônica de linfocitos B: un modelo personalizado de valoración clínica y molecular. Rev. Hematol. Mex. 15:103-121, 2014.

VASCONCELOS, Y. Marcadores de prognóstico na leucemia linfocítica crônica. Rev. Bras. Hematol. Hemoter. Goiânia, v.27 n.4, p. 247-252, 2005.

WINKLER, D.; et al. Protein expression analysis of chromosome 12 candidate genes in chronic lymphocytic leukemia (CLL). Leukemia 19 (7), 1211-1215, 2005. 


\section{(A) CONAGEN

XIE, Y.; DAVIES, S.M.; XIANG, Y.; et al. Thrends in luekemia incidence and survival in the United States (1973- 1998). I Cancer 2003; 97: 2.229-35.

YAMAMOTO, M.; FIGUEIREDO, V. L. P. Epidemiologia da leucemia linfocítica crônica e leucemia linfocítica crônica familiar. Rev. Bras. Hematol. Hemater. v. 27 n. 4, p. 229-232, 2005.

ZENZ, T.; et al. From pathogenesis to treatment of chronic lymphocytic leukaemia. Nature Reviews, Gremany, v. 10, p. 37-50. 2010. 